> La flore intestinale est aujourd'hui considérée comme un organe à part entière, qui joue un rôle clé dans le métabolisme énergétique. La flore peut en effet augmenter la rentabilité énergétique des aliments ingérés qui ont échappé à la digestion dans la partie haute de l'intestin, via leur fermentation. Par ailleurs, le flux des acides gras et leur stockage adipocytaire sont contrôlés - notamment via le facteur FIAF (fastinginduced adipocyte factor), dont l'expression est tributaire de la présence de la flore intestinale. Des modifications de l'écosystème bactérien de l'intestin pourraient être impliquées dans le développement des altérations métaboliques liées au diabète de type 2 et à l'obésité. Des données expérimentales démontrent qu'une alimentation hyperlipidique modifie la composition de la flore intestinale, en diminuant notamment les bifidobactéries, avec pour conséquence une augmentation de l'absorption et des taux plasmatiques de lipopolysaccharide, qui participent à leur tour au déclenchement et au développement de l'inflammation, de l'insulino-résistance et du développement de masse grasse. Rééquilibrer ou modifier la composition de la flore intestinale pourrait constituer une voie thérapeutique ou préventive susceptible de réduire l'impact d'une alimentation déséquilibrée sur le développement du syndrome métabolique. <

\section{Implication de la flore intestinale dans le métabolisme énergétique}

Nathalie M. Delzenne, Patrice D. Cani

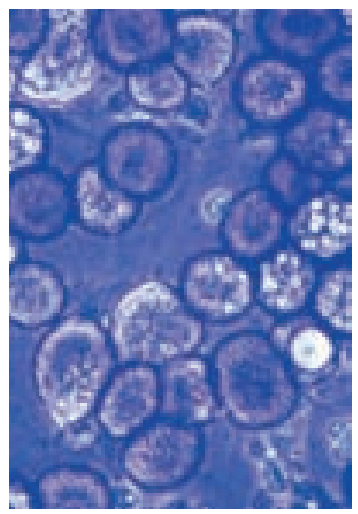

Université catholique de Louvain, Unité de Pharmacocinétique, Métabolisme, Nutrition et Toxicologie, Bruxelles, Belgique. UCL, Unit PMNT-7369, Avenue $\varepsilon$. Mounier, 73/69, B-1200 Bruxelles, Belgique.

nathalie.delzenne@uclouvain.be à un «stress nutritionnel » (régime riche en lipides), seuls certains individus développeront une obésité et une hyperglycémie, tandis que d'autres seront moins sensibles aux altérations métaboliques liées à la diète $[1,2]$. Même si la susceptibilité individuelle peut être liée au génome, un nouveau facteur «environnemental » étroitement associé à l'individu, et qui le caractérise dès la naissance, semble impliqué dans le contrôle du poids corporel et de l'homéostasie énergétique : la flore intestinale. Au cours de cette synthèse nous tenterons d'éclaircir les premières études expérimentales qui ont permis de lever un coin du voile sur le rôle de la flore intestinale dans le contrôle du métabolisme énergétique.

\section{La flore intestinale de l'homme : un jardin secret}

Le tractus gastro-intestinal - principalement le côlon de l'homme abrite environ $10^{14}$ cellules bactériennes issues de près de 1000 espèces, ce qui dépasse largement toutes les autres communautés bactériennes associées au corps humain [3]. Pour cette raison, l'homme est parfois décrit comme un «métagénome», composé de l'ensemble des gènes du génome humain d'une part, et du microbiome bactérien d'autre part [4]. Pris dans son entièreté, le microbiome comporte 100 fois plus de gènes que le génome humain [5]. La grande majorité des espèces bactériennes présentes 
dans l'intestin ne sont pas détectées dans les études faisant appel à des techniques classiques basées sur la culture de bactéries. Plus récemment, les informations concernant la diversité et les aspects fonctionnels des microorganismes présents au sein du biotope intestinal ont pu être révélées grâce à des techniques d'analyse fondées sur l'étude de I'ARN16S des bactéries [5-7]. La plupart des études mécanistiques et métagénomiques ont été réalisées dans des modèles animaux, qui ont permis de démontrer que les micro-organismes qui colonisent l'intestin fournissent des attributs génétiques et métaboliques utiles, notamment pour faire face aux facteurs environnementaux auxquels nous sommes confrontés. Citons, parmi les rôles clés de la flore intestinale: la défense face aux micro-organismes pathogènes, le renforcement de l'immunité (via un ensemble de signaux ou métabolites, jouant un rôle in situ mais également systémique), le développement des microvillosités intestinales, la fermentation de nutriments qui ont échappé à la digestion dans la partie haute de l'intestin (fibres alimentaires, amidon résistant, oligosaccharides), le métabolisme anaérobie de peptides ou de protéines, la bio-transformation des acides biliaires, ou encore la synthèse de certaines vitamines (vitamines $B 12$ et $K$ ) [8]. La flore intestinale peut dès lors être considérée comme un véritable «organe extériorisé » dont les fonctions contribuent à l’homéostasie métabolique créant une véritable symbiose avec l'or-

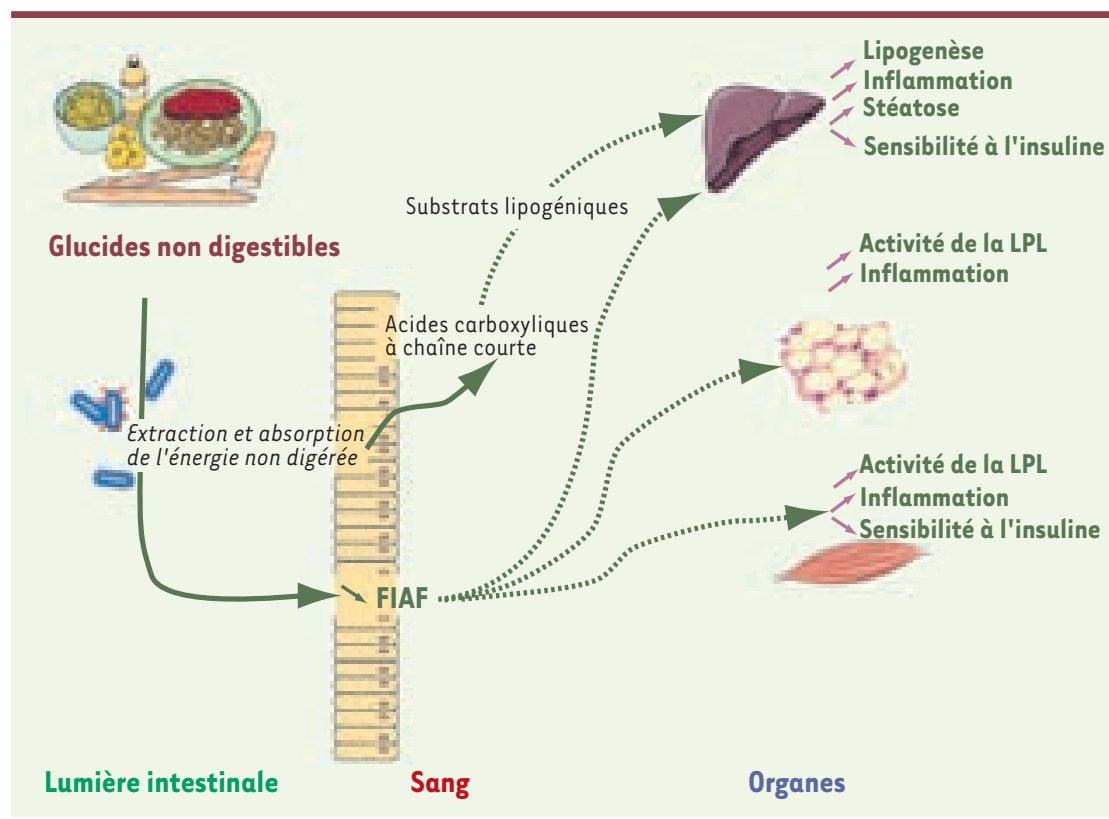

Figure 1. Rôle de la flore intestinale dans le développement des maladies métaboliques associées à l'obésité. Théorie de la rentabilité énergétique de l'aliment et du FIAF. L'énergie contenue dans les glucides non digestibles - échappant à la digestion par les enzymes du tractus digestif supérieur- peut être rendue disponible pour l'hôte grâce à l'intervention des bactéries intestinales, qui les fermentent en acides carboxyliques à chaînes courtes. Ces derniers peuvent être utilisés comme substrats lipogéniques et gluconéogéniques par le foie. Par ailleurs, la flore intestinale régule l'expression de la protéine FIAF - la diminution de l'expression de FIAF augmentant l'activité de la lipoprotéine lipase, permettant aux acides gras d'être captés par les tissus périphériques pour être stockés ou oxydés. FIAF : fasting induced adipose factor; LPL : lipoprotéine lipase. ganisme hôte. Les micro-organismes présents dans le côlon possèdent les caractéristiques génomiques qui leur confèrent la capacité d'utiliser les nutriments «providentiels » qui ont échappé à la digestion dans la partie haute l'intestin. En retour, elles produisent des métabolites (acides carboxyliques à chaîne courte) qui, une fois absorbés par les cellules intestinales, contribuent en tant que substrat et régulateur métabolique de l'hôte [9].

\section{Comment la flore peut-elle modifier} Les bactéries intestinales régulent

le stockage d'énergie chez leur hôte

Les fonctions biologiques contrôlées par la flore intestinale semblent être en relation avec l'efficacité des bactéries à récupérer l'énergie ingérée mais non digérée par l'hôte. L'homéostasie du poids corporel dépend de mécanismes finement régulés au cours du temps. Un excès énergétique de moins de $1 \%$ par rapport aux besoins énergétiques pourrait être suffisant pour entraîner une augmentation du poids corporel sur une période équivalente à plusieurs années [10]. Par conséquent, tous les mécanismes impliqués dans la disponibilité de l'énergie provenant de l'alimentation pourraient contribuer à l'équilibre du poids corporel. Plusieurs études américaines émanant du laboratoire de J. Gordon ont suggéré l'implication de la flore intestinale dans la régulation de l'homéostasie énergétique [11]. Il y a quelques années, F. Backhed et ses collaborateurs ont mis en évidence le fait suivant: de jeunes souris élevées de façon classique possèdent - malgré un apport alimentaire en calories moins important - une masse adipeuse plus développée (+ $40 \%$ ) que celle des souris exemptes de flore intestinale (souris axéniques) [12]. Dans le même ordre d'idée, les auteurs ont démontré que les souris dépourvues de flore intestinale à la naissance, puis colonisées avec une flore intestinale émanant de souris normales, développent davantage de masse grasse (environ $+60 \%$ ) et présentent une diminution de la sensibilité à l'insuline deux semaines après l'instauration de la flore. Plusieurs mécanismes sont évoqués, qui pourraient contribuer à l'augmentation 
du poids corporel en présence des bactéries de la flore: une augmentation de l'absorption du glucose par l'intestin; une augmentation de la disponibilité de l'énergie via la fermentation en acides carboxyliques à chaîne courte des composés non digestibles de l'alimentation (Figure I); et /ou une augmentation de l'insulinémie qui contribue à l'anabolisme [13]. De façon intéressante, le fait de coloniser l'intestin des souris axéniques avec une flore conventionnelle provoque également une augmentation généralisée de l'activité de l'enzyme lipoprotéine lipase (LPL) (Figure 1). Cette dernière catalyse la libération des acides gras à partir des triglycérides associés aux lipoprotéines circulantes, permettant ainsi aux acides gras d'être captés par le muscle ou le tissu adipeux. Les auteurs ont proposé que cette augmentation est la conséquence de la suppression de l'expression d'un facteur dérivé de l'intestin: le fasting-induced adipose factor (FIAF), un inhibiteur de l'activité de la LPL. La diminution de l'expression de FIAF observée chez les souris colonisées, participerait à l'accumulation de lipides dans le tissu adipeux. Ces expériences ont démontré pour la première fois que les bactéries intestinales pouvaient réguler le stockage d'énergie chez l'hôte qu'elles colonisent [12] (Figure 1).

Importance des modifications qualitatives de la flore Ley et ses collaborateurs ont démontré, chez le rongeur, que l'obésité pouvait être associée à des modifications qualitatives de la flore intestinale. Après avoir caractérisé plus de 5000 séquences d'ARN16S de bactéries intestinales de souris obèses $o b / o b$ et de leurs congénères sauvages, les auteurs ont mis en évidence que les animaux obèses possédaient deux fois moins de Bacteroidetes et une augmentation proportionnelle des Firmicutes, ces deux grands phylums bactériens représentant plus de $85 \%$ de la microflore intestinale. Ces mêmes auteurs ont comparé la flore intestinale d'humains obèses et minces. Afin d'analyser la relation entre l'écologie de la flore microbienne et la masse adipeuse de l'homme, ils ont étudié 12 obèses volontaires soumis à un régime hypocalorique appauvri en lipides ou en glucides. Les résultats montrent qu'avant le traitement, à l'instar des observations réalisées chez l'animal, les sujets obèses possédaient moins de Bacteroidetes et plus de Firmicutes que les sujets minces [14]. Chez les obèses, la perte de poids a permis de restaurer un profil bactérien similaire à celui des sujets minces et ce indépendamment du type de régime instauré. Les auteurs démontrent qu'il existe une corrélation positive entre le poids corporel et l'augmentation des Bacteroidetes. Ces données suggèrent que le poids corporel per se, indépendamment de la nature du régime alimentaire, intervient dans la gestion du profil des bactéries intestinales. Si les résultats obtenus à la fois chez l'homme et chez l'animal, suggèrent que l'obésité altère la composition de la flore intestinale, cela ne prouve nullement que la différence relative de distribution bactérienne explique - à son tour - la différence de poids corporel. Des données expérimentales étayent cette hypothèse, selon laquelle les caractéristiques de la flore ellemême participent à un changement de comportement alimentaire et métabolique. Les chercheurs ont réalisé une expérience clé au cours de laquelle la flore de souris obèses $o b / o b$ a été transférée à des souris minces axéniques. Les auteurs ont observé que le poids corporel des souris minces colonisées avec la flore de souris obèses augmentait dès la deuxième semaine [4]. L'augmentation de masse grasse observée chez les souris axéniques minces colonisées avec la flore intestinale de souris obèses est davantage tributaire de l'augmentation - quoique modeste - des ingestats que des calories récupérées lors de la fermentation des glucides non digestibles. Des arguments expérimentaux d'une autre nature suggèrent que la fermentation de glucides non digestibles n'augmente pas la masse grasse, mais, au contraire, contribue à réduire les altérations métaboliques associées à l'obésité. Ainsi, notre équipe et d'autres auteurs ont montré qu'une alimentation riche en fibres «fermentescibles » non digestibles (fructanes) diminuait le poids corporel, le développement de la masse adipeuse et la sévérité du diabète dans plusieurs modèles animaux [15-19]. Ces fibres alimentaires sont totalement fermentées dans le côlon, permettant aux bactéries utilisant préférentiellement ces composés comme source énergétique - en l'occurrence les bifidobactéries - de se développer. Comme nous l'illustrerons plus loin, ceci renforce l'idée suivante: une modulation ciblée de certaines espèces bactériennes pourrait également intervenir dans le développement de l'obésité. Les souris axéniques résistent également à l'obésité induite par un régime riche en lipides [20]. Les auteurs ont observé que les souris qui possèdent une flore indigène, nourries avec un régime de type western diet (riche en graisses et en sucres), gagnaient davantage de poids et de tissu adipeux, et augmentaient leur glycémie et leur insulinémie par rapport à leurs congénères axéniques soumises au même régime. De façon étonnante et opposée aux observations faites chez les souris axéniques nourries avec une diète normale, les souris axéniques soumises à la diète western consommaient et excrétaient la même quantité d'énergie que les souris qui possédaient une flore. Ces données suggèrent donc qu'il existe un facteur dépendant des bactéries intestinales, qui pourrait promouvoir la réponse métabolique (en terme de développement de masse grasse) à un régime riche en lipides.

\section{L'inflammation d'origine bactérienne pourrait-elle être le facteur responsable du développement de l'obésité et du diabète?}

L'ingestion d'une alimentation riche en graisses se caractérise par le développement d'un diabète de type 2 et d'une obésité, eux-mêmes étroitement liés à un état inflammatoire de faible intensité [21]. Nous avons récemment exploré comment un facteur associé aux bactéries 
pouvait être responsable du développement de l'obésité et du diabète au cours d'une alimentation hyperlipidique. Nous avons découvert que le lipopolysaccharide issu des bactéries Gram-négative présentes dans l'intestin pourrait constituer le candidat idéal. Le lipopolysaccharide est produit de façon continue dans l'intestin suite à la lyse des bactéries Gram-négative et est physiologiquement absorbé puis transporté de l'intestin vers les tissus cibles par un mécanisme dépendant des lipoprotéines [22, 23]. De plus, le lipopolysaccharide stimule la sécrétion de cytokines pro-inflammatoires lorsqu'il se lie à son complexe CD14/TLR4 présent à la surface des cellules immunitaires [24]. En accord avec ce concept, nous avons montré que l'ingestion d'une diète hyperlipidique modifiait de façon importante les membres dominants des populations de bactéries de l'intestin de la souris. Nous avons observé une diminution drastique du nombre de bifidobactéries, mais aussi des bactéries du groupe des $\varepsilon$ u. Rectale/Cl. Coccoides et des bactéries intestinales associées aux Bacteroides. Cette modification majeure de la flore intestinale est associée à une augmentation de l'endotoxémie, du poids corporel, du contenu en triglycérides dans le foie, du diabète et du tonus inflammatoire [25] (Figure 2). Afin de démontrer que le lipopolysaccharide agit comme facteur stimulant du déclenchement des désordres métaboliques, des souris invalidées pour le récepteur CD14 ont été soumises à un régime hyperlipidique. Nous avons pu observer que, en l'absence du récepteur au lipopolysaccharide, les souris résistaient au développement de tous les stigmates métaboliques induits par le traitement alimentaire riche en gras. En accord avec nos découvertes, une étude récente a démontré que les souris $o b / o b$ et $d b / d b$ présentaient une endotoxémie élevée [26]. De plus, un traitement avec de la polymyxine $B$, qui élimine spécifiquement les bactéries Gram-négative et inhibe également le lipopolysaccharide, permet de diminuer la stéatose hépatique. En

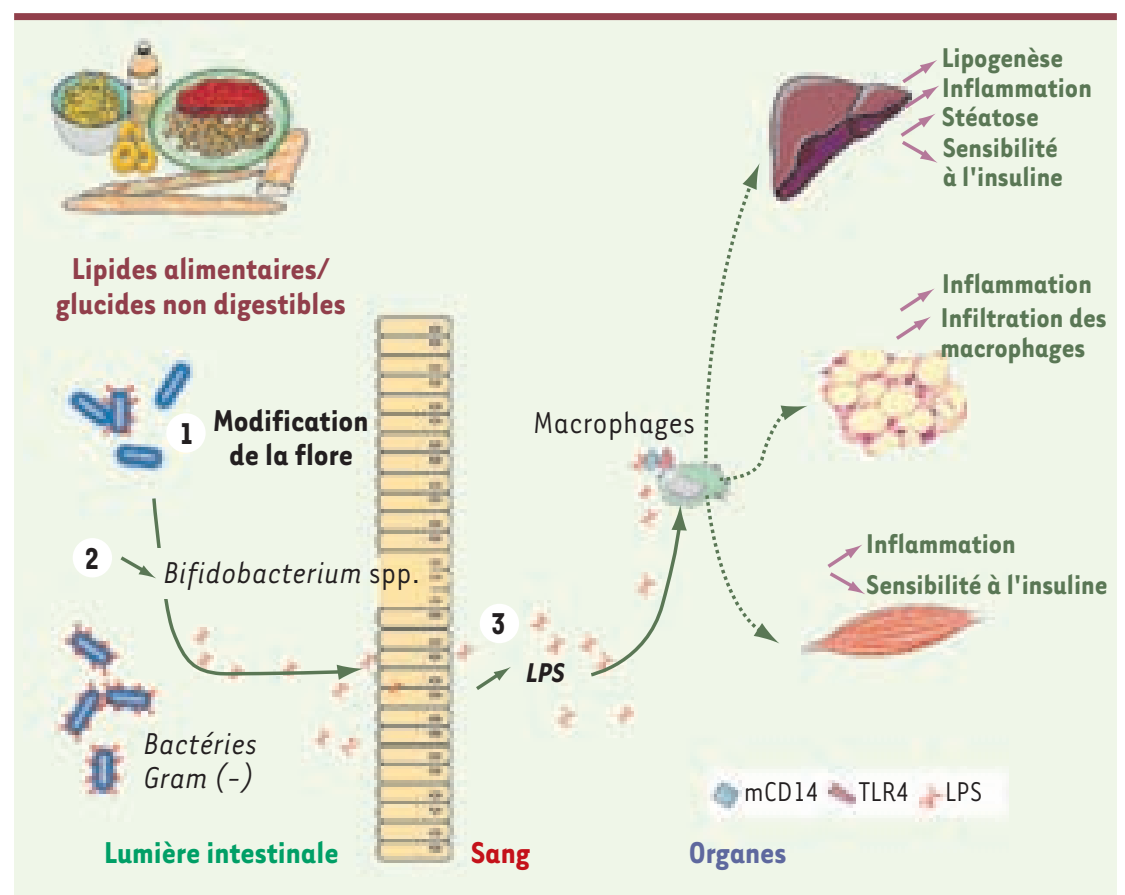

ce qui concerne les données chez l'homme, Creely et ses collaborateurs ont apporté des arguments en faveur de I'hypothèse selon laquelle le lipopolysaccharide pourrait agir comme facteur associé aux bactéries et être impliqué dans le développement du diabète de type 2 et de l'obésité. Ils ont observé que les taux de lipopolysaccharides plasmatiques étaient significativement augmentés chez des individus présentant un diabète de type 2. De plus, l'insulinémie à jeun était significativement corrélée avec l'endotoxémie dans la totalité de la population des individus non diabétiques et cette corrélation restait présente même en contrôlant le sexe, l'âge et l'indice de masse corporelle [27].

Toutes ces études suggèrent fortement le rôle potentiel d'un facteur dérivé de la flore intestinale (le lipopolysaccharide) dans la pathogénie du diabète associé à l'obésité.

\section{Quels sont les médiateurs métaboliques tributaires d'une modification sélective de la flore intestinale?}

Parmi les outils utilisés pour modifier la flore intestinale, les prébiotiques et les probiotiques font parties des plus importants. Un prébiotique est: «un nutriment non digéré et fermenté de façon sélective par des bactéries (bifidobactéries, lactobacilles) entraînant des modifications à la fois de la composition de la flore intestinale mais aussi de son activité, ce qui se répercute de façon bénéfique sur le bien-être et la santé de l'hôte » [28]. Les probiotiques contiennent des bactéries vivantes administrées oralement en quantité adéquate afin de coloniser le côlon. Les fructo-oligosaccharides de

Figure 2. Modification de la flore intestinale après ingestion d'une alimentation hyperlipidique et mécanismes associés au développement de l'inflammation, du diabète et de l'obésité. La théorie du lipopolysaccharide (LPS). L'ingestion d'une alimentation riche en lipides modifie la composition de la flore intestinale (1), avec plus particulièrement une diminution des bifidobactéries (2). Cette modification de la flore intestinale est associée à une augmentation des taux de LPS plasmatique (3). Le LPS après liaison à son récepteur complexe CD14/TLR4, stimule la synthèse et la sécrétion de cytokines proinflammatoires qui participent au développement de l'insulinorésistance. LPS: lipopolysaccharides. 


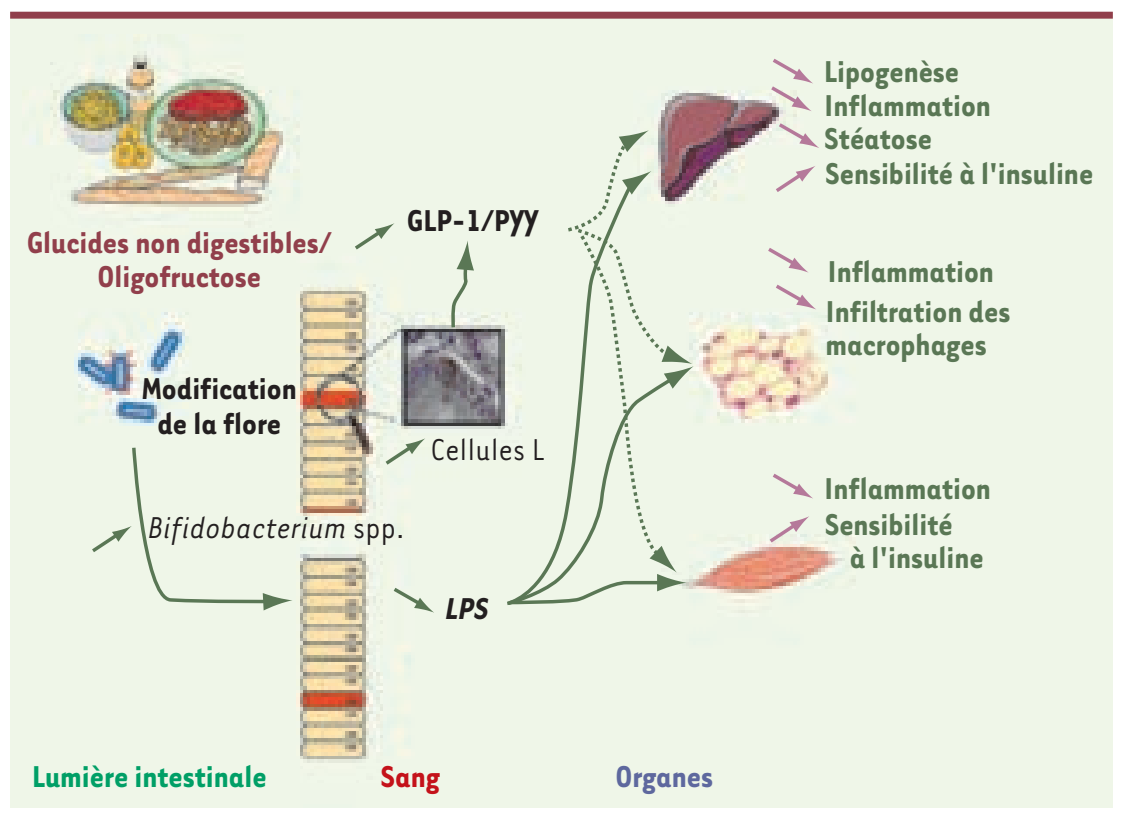

Figure 3. Modification de la flore intestinale par les prébiotiques et effets physiologiques. Rôle des bifidobactéries, et des peptides gastro-intestinaux. L'ingestion de fibres fermentescibles de types prébiotiques augmente de façon significative et sélective le contenu en bifidobactéries dans I'intestin. Ceci s'accompagne d'une baisse des taux de LPS plasmatiques et d'une diminution de l'inflammation. Par ailleurs, la fermentation des prébiotiques augmente le nombre de cellules $L$ intestinales et leurs produits de sécrétion - GLP- 1 et Pyy - tout deux impliqués dans la régulation de l'homéostasie glucidique et de la satiété. LPS : lipopolysaccharides; GLP-1: glucagon-like peptide-1; Pyy : peptide Yy.

type fructanes ont clairement un rôle de prébiotiques. Ces fibres alimentaires fermentescibles sont capables d'augmenter de façon significative les bactéries du genre Bifidobacterium spp. et Lactobacillus spp. chez l'homme et l'animal. Les bifidobactéries sont capables de réduire le contenu intestinal en lipopolysaccharides et d'améliorer l'intégrité de la muqueuse intestinale. Nos derniers travaux ont permis de confirmer l'origine et le rôle de la modification sélective de la flore intestinale dans le développement des désordres métaboliques induits par une diète hyperlipidique. En effet, nous avons montré que restaurer le nombre de bifidobactéries de souris ayant reçu une diète hyperlipidique à l'aide de prébiotiques permettait de contrôler l'augmentation des taux de lipopolysaccharides plasmatiques (Figure 2). L'augmentation sélective des bifidobactéries est corrélée à une normalisation du tonus inflammatoire, une amélioration de la tolérance au glucose et de la sécrétion d'insuline induite par le glucose [29]. Donc ces dernières données démontrent que la flore intestinale pourrait être une cible intéressante afin de maintenir ou restaurer des fonctions métaboliques normales.

Une modulation de la flore intestinale en faveur des bifidobactéries pourrait aussi être associée à une régulation de la production de peptides intestinaux impliqués dans la gestion de l'appétit, de la sécrétion/sensibilité à l'insuline, de la glycémie et du poids corporel. En effet, l'ingestion de prébiotiques de type fructanes augmente la production endogène de peptides comme le glucagon-like peptide-1 (GLP-1), le glucose-dependent insulinotropic peptide (GIP) et le peptide Yy, et diminue les taux plasmatiques d'une hormone orexigène la ghréline [15-18, 30, 31] (Figure 3). Les produits du catabolisme bactérien des fructanes - tels que le butyrate interviennent dans l'augmentation de la production de GLP-1 par les cellules endocrines du côlon. L'impact d'autres métabolites bactériens sur les centres périphériques et hypothalamiques de contrôle de l'appétit pourrait constituer une voie intéressante d'investigation. Nous ne pouvons pas exclure qu'il puisse exister un lien entre le facteur inflammatoire dérivé de la flore intestinale, le lipopolysaccharide et la production endogène de peptides gastro-intestinaux tel que le GLP-1. L'amélioration des paramètres inflammatoires associés à la fois aux modifications de la flore intestinale et aux désordres métaboliques mérite d'être étudiée plus particulièrement.

\section{Conclusion}

Le fait que la flore intestinale soit reconnue comme un acteur important de l'homéostasie énergétique invite la communauté scientifique à reconsidérer cet «organe » dans le contexte de l'obésité. La validation de méthodes moléculaires permettant l'analyse quantitative des bactéries a permis des avancées considérables dans la connaissance de la composition de la flore intestinale ces cinq dernières années. La différence de composition de la flore intestinale entre des individus sains ou obèses et diabétiques de type 2 est une découverte importante qui permet d'établir la manière dont la flore dialogue avec l'hôte dans le contexte de la physiopathologie des maladies métaboliques. II semble que plusieurs mécanismes à la fois différents mais complémentaires puissent être proposés. Premièrement, la flore pourrait être capable d'augmenter la rentabilité énergétique des aliments ingérés en extrayant davantage l'énergie qu'ils contiennent pour la redistribuer à l'hôte; deuxièmement, la flore intestinale pourrait contrôler l'expression de facteurs impliqués dans le flux des acides gras et dans leur stockage adipocytaire (théorie du FIAF); et troisièmement, un régime hyperlipidique entraînerait des altérations de la composition de la flore intestinale, notamment 
une diminution des bifidobactéries, une augmentation de l'absorption et des taux plasmatiques de lipopolysaccharides déclenchant le développement de l'inflammation, de l'insulino-résistance et du diabète de type 2 associé à l'obésité. Néanmoins un certain nombre de questions restent non élucidées. Pourquoi et comment la flore intestinale est-elle modifiée au cours de l'obésité ? À ce jour - à l'instar de l'histoire de la poule et de l'œuf - nous ne pouvons clairement affirmer que c'est la flore intestinale qui déclenche l'obésité, ou l'obésité qui provoque les altérations de la flore. Cependant, quel que soit le profil de la flore intestinale qui, rappelons-le, est acquis lors de la naissance, et quel que soit l'état de la flore à l'âge adulte, des interventions nutritionnelles peuvent rééquilibrer la flore intestinale en faveur d'un profil présentant les caractéristiques d'un sujet non obèse, allant de pair avec une amélioration de l'état métabolique. À cet égard, la présence d'une flore faisant la part belle aux bifidobactéries semble intéressante. Pouvons-nous proposer des stratégies visant à augmenter les bifidobactéries dans l'intestin, via les prébiotiques ou les probiotiques dans le contexte de l'obésité ? Les moyens permettant de modifier la composition de la flore intestinale pourraient constituer un outil thérapeutique ou préventif permettant de réduire l'impact d'une alimentation riche en lipides et pauvre en fibres alimentaires sur le développement des maladies métaboliques. $\diamond$

\section{SUMMARY}

\section{Gut microflora is a key player}

in host energy homeostasis

Gut microflora is now considered as a key organ invovled in host energy homeostasis. Recent data suggest that the alterations of the gut bacteria ecosystem could contribute to the development of metabolic disorders such as type 2 diabetes and obesity. First, gut microflora may increase energy efficiency of non digested food via the fermentation, thus providing more energy to the host. Secondly, fatty acids flux and storage in the adipose tissue is under the control of the fasting-induced adipocyte factor FIAF, which expression depends on gut microflora. Third, high-fat diet feeding changes gut bacteria profile, leading to a drop in bifidobacteria content, which correlates with a higher LPS plasma levels, thereby participating to the onset of inflammation, insulin resistance and type 2 diabetes associated with obesity. Changing gut microflora composition could be a useful tool to prevent or to treat high-fat/low fibres diet-induced metabolic syndrome. $\diamond$

\section{RÉFÉRENCES}

1. Tappy L. Metabolic consequences of overfeeding in humans. Curr Opin Clin Nutr Metab Care $2004 ; 7: 623-8$.

2. Levin BE, Keesey RE. Defense of differing body weight set points in diet-induced obese and resistant rats. Am J Physiol $1998 ; 274$ : R412-R419.

3. Savage DC. Microbial ecology of the gastrointestinal tract. Annu Rev Microbiol 1977 ; $31: 107-33$.

4. Turnbaugh PJ, Ley RE, Mahowald MA, et al. An obesity-associated gut microbiome with increased capacity for energy harvest. Nature $2006 ; 444$ : 1027-31.

5. Xu J, Mahowald MA, Ley RE, et al. Evolution of Symbiotic Bacteria in the Distal Human Intestine. PLoS Biol 2007 ; 5 : el56.

6. Ley RE, Peterson DA, Gordon JI. Ecological and evolutionary forces shaping microbial diversity in the human intestine. Cell $2006 ; 124: 837-48$.

7. Gill SR, Pop M, Deboy RT, et al. Metagenomic analysis of the human distal gut microbiome. Science 2006 ; 312 : 1355-9.

8. Nicholson JK, Holmes $\varepsilon$, Wilson ID. Gut microorganisms, mammalian metabolism and personalized health care. Nat Rev Microbiol 2005 ; 3 : 431-8.

9. Wong JM, De SR, Kendall CW, et al. Colonic health: fermentation and short chain fatty acids. J Clin Gastroenterol $2006 ; 40: 235-43$.

10. Hill J0. Understanding and addressing the epidemic of obesity: an energy balance perspective. Endocr Rev $2006 ; 27: 750-61$.

11. Servin AL. Ça «chat » entre la microflore intestinale et l'hôte. Med Sci (Paris) 2007 ; $23: 229-30$.

12. Backhed F, Ding $H$, Wang $T$, et al. The gut microbiota as an environmental factor that regulates fat storage. Proc Natl Acad Sci USA 2004 ; 101 : 15718-23.

13. Foufelle $F$, Hegarty $B$, Bobard $A$, et al. Un nouveau rôle de l'insuline dans la régulation du métabolisme glucido-lipidique hépatique. Med Sci (Paris) 2005 ; 21 : 569-71.

14. Ley RE, Turnbaugh PJ, Klein $S$, et al. Microbial ecology: human gut microbes associated with obesity. Nature $2006 ; 444: 1022-3$.

15. Cani PD, Neyrinck AM, Maton N, et al. Oligofructose promotes satiety in rats fed a high-fat diet: involvement of glucagon-like Peptide-1. Obes Res 2005 ; 13 : 1000-7.

16. Cani PD, Daubioul CA, Reusens B, et al. Involvement of endogenous glucagon-like peptide1 (7-36) amide on glycaemia-lowering effect of oligofructose in streptozotocin-treated rats. $J$ Endocrinol $2005 ; 185: 457-65$.

17. Cani PD, Knauf C, Iglesias MA, et al. Improvement of glucose tolerance and hepatic insulin sensitivity by oligofructose requires a functional glucagon-like peptide 1 receptor. Diabetes $2006 ; 55: 1484-90$.

18. Cani $P D$, Joly $\varepsilon$, Horsmans $Y$, et al. Oligofructose promotes satiety in healthy human: a pilot study. Eur J Clin Nutr $2006 ; 60: 567-72$.

19. Keenan MJ, Zhou J, McCutcheon KL, et al. Effects of resistant starch, a non-digestible fermentable fiber, on reducing body fat. Obesity (Silver Spring) 2006 ; 14 : 1523-34.

20. Backhed F, Manchester JK, Semenkovich CF, et al. Mechanisms underlying the resistance to diet-induced obesity in germ-free mice. Proc Natl Acad Sci USA 2007 ; 104 : 979-84.

21. Hotamisligil GS. Inflammation and metabolic disorders. Nature $2006 ; 444: 860-7$.

22. Neal MD, Leaphart C, Levy R, et al. Enterocyte TLR4 mediates phagocytosis and translocation of bacteria across the intestinal barrier. J Immunol 2006 ; 176:3070-9.

23. Vreugdenhil AC, Rousseau CH, Hartung T et al. Lipopolysaccharide (LPS)-binding protein mediates LPS detoxification by chylomicrons. J Immunol 2003 ; 170 : 1399-405.

24. Wright SD, Ramos RA, Tobias PS, et al. CD14, a receptor for complexes of lipopolysaccharide (LPS) and LPS binding protein. Science $1990 ; 249: 1431-3$.

25. Cani PD, Amar J, Iglesias MA, et al. Metabolic endotoxemia initiates obesity and insulin resistance. Diabetes $2007 ; 56: 1761-72$.

26. Brun P, Castagliuolo I, Leo VD, et al. Increased intestinal permeability in obese mice: new evidence in the pathogenesis of nonalcoholic steatohepatitis. Am J Physiol Gastrointest Liver Physiol 2007 ; 292 : G518-25.

27. Creely SJ, McTernan PG, Kusminski CM, et al. Lipopolysaccharide activates an innate immune system response in human adipose tissue in obesity and type 2 diabetes. Am J Physiol Endocrinol Metab 2007 ; 292 : $8740-7$.

28. Gibson GR, Roberfroid MB. Dietary modulation of the human colonic microbiota: introducing the concept of prebiotics. J Nutr 1995 ; $125: 1401-12$.

29. Cani PD, Neyrinck AM, Fava F, et al. Selective increases of bifidobacteria in gut microflora improve high-fat-diet-induced diabetes in mice through a mechanism associated with endotoxaemia. Diabetologia $2007 ; 50: 2374-83$.

30. Cani PD, Dewever C, Delzenne NM. Inulin-type fructans modulate gastrointestinal peptides involved in appetite regulation (glucagon-like peptide-1 and ghrelin) in rats. Br J Nutr 2004 ; $92: 521-6$.

31. Delzenne NM, Cani PD, Daubioul C, et al. Impact of inulin and oligofructose on gastrointestinal peptides. BrJ Nutr 2005 ; 93 (suppl 1) : S157-61.

\section{TIRÉS À PART}

N. Delzenne 